\title{
Topological Entropy: A Lagrangian Measure of the State of the Free Atmosphere
}

\author{
TÍMEA HASZPRA AND TAMÁS TÉL \\ MTA-ELTE Research Group in Theoretical Physics, Eötvös Loránd University, Budapest, Hungary
}

(Manuscript received 25 February 2013, in final form 31 May 2013)

\begin{abstract}
Topological entropy is shown to be a useful characteristic of the state of the free atmosphere. It can be determined as the stretching rate of a line segment of tracer particles in the atmosphere over a time span of about 10 days. Besides case studies, the seasonal distribution of the average topological entropy is determined in several geographical locations. The largest topological entropies appear in the mid- and high latitudes, especially in winter, owing to the greater temperature gradient between the pole and the equator and the more intense stirring and shearing effects of cyclones. The smallest values can be found in the trade wind belt. The local value of the topological entropy is a measure of the chaoticity of the state of the atmosphere and of how rapidly pollutants and contaminants spread from a given location.
\end{abstract}

\section{Introduction}

The advection of tracers and particles in the atmosphere and the characterization of this process have been investigated in many different ways. Several studies characterize the advection of tracers by means of various Lagrangian quantities, like Lyapunov exponents (Pierrehumbert and Yang 1993; Bowman 1993; von Hardenberg et al. 2000; Mizuta and Yoden 2001), fractal dimensions (Pierrehumbert and Yang 1993; Yang and Pierrehumbert 1994; von Hardenberg et al. 2000; Mizuta and Yoden 2001), and diffusion coefficients (Bowman 1993; von Hardenberg et al. 2000; Huber et al. 2001).

Here we propose the use of another quantity, well known in dynamical systems theory (Ott 1993; Tél and Gruiz 2006), which turns out to have a very natural meaning in the context of dispersion-namely, the stretching rate of material lines. The numerical determination of this quantity proves to be easier than that of many other Lagrangian measures. Being basically a concept used in chaos theory, topological entropy can be used in situations when the tracer dynamics can be well described in terms of an ordinary differential equation with the wind field as input data. This is the

Corresponding author address: Tímea Haszpra, Institute of Theoretical Physics and MTA-ELTE Research Group in Theoretical Physics, Eötvös Loránd University, Pázmány P. s. 1/A, Budapest H-1117, Hungary.

E-mail: hatimi@caesar.elte.hu case on large scales in the free atmosphere where the effect of turbulent diffusion is weak and dry and wet deposition are negligible (i.e., when tracers remain outside the boundary layer). In the simulations presented in the bulk of the paper, for simplicity, we neglect these processes also in the boundary layer where a small fraction of the particles enter.

In this paper passive tracers are tracked in reanalysis wind fields. In a first approximation passive tracers are advected along isentropic surfaces (e.g., Stohl 1998). In our study we calculate the 3D trajectory of the tracers in order to demonstrate that determining the topological entropy does not imply any complication even in fully $3 \mathrm{D}$ wind fields.

We initialize line segments of particles in the free atmosphere, and in each time step calculate the length of the filament. This increases typically in an exponential manner in time. It is the rate of this exponential stretching of the filament length that provides the topological entropy. The numerical determination of this is much easier than, say, that of the Lyapunov exponent. Since the initial length need not be infinitesimally small, neighboring particles need not remain close to each other; on the contrary, the length of the material line can reach several thousands of kilometers. The topological entropy is known to be an upper bound to the Lyapunov exponent. Typical values are found to be on the order of 0.6 day $^{-1}$ in mid- and high latitudes. This implies that the length is stretched by a factor of 400 over 10 days and a factor of more than $6 \times 10^{7}$ over a month. 
The topological entropy characteristic to the time span of about 10 days turns out to depend on the actual wind field; therefore, the determination of a geographical and seasonal distribution of the topological entropy appeared to be reasonable. The result is that different values show up in the range of $0.3-0.8 \mathrm{day}^{-1}$. This range seems to be not very large, but being in the exponent, it implies length ratios of about 150 over 10 days and $3 \times 10^{6}$ over a month.

The paper is organized as follows. In section 2 we provide a brief overview of the concept of the topological entropy and point out what way is the most efficient for its determination in an atmospheric context. Section 3 presents the equations of the motion for tracer particles advected in a given wind field and the data and numerical methods used in the simulations. In section 4 a case study is presented to demonstrate how the method works in detail. The results concerning the geographical distribution of the topological entropy in different periods of a year are given in section 5. Local variances are investigated in section 6 where we show that topological entropy can be considered as a measure of the spreading of large-scale contaminations. Section 7 contains a discussion and outlook.

\section{Topological entropy}

When the advection of a tracer particle is considered in a given wind field $\mathbf{v}(\mathbf{r}, t)$ of the free atmosphere, on large scales, diffusion turns out to be negligible. An orderof-magnitude estimate reveals that the average distance $D$ over which a pointlike initial concentration spreads in a time interval of $T=10$ days in the presence of a typical horizontal turbulent diffusion of $K_{h}=10-10^{3} \mathrm{~m}^{2} \mathrm{~s}^{-1}$ is $D \approx \sqrt{K_{h} T} \approx 3-30 \mathrm{~km}$. On horizontal scales larger than $30 \mathrm{~km}$, which is much smaller than the advection length scale, typically of $1000 \mathrm{~km}$, the equation of motion is an ordinary differential equation (see section 3 ) for the path of a particle. The dispersion problem therefore belongs to the class of low-dimensional dynamical systems [for an introductory text, see Tél and Gruiz (2006)], and the appearance of chaos is very likely in such cases.

In the best-known examples of chaos, the driving is typically periodic in time. This is different in our problem, where the motion of the particle in the turbulent state of the atmosphere is driven by a temporally irregular external forcing. Although more complicated than traditional cases, the dispersion problem under the conditions mentioned above provides an example of chaotic advection (Aref 1985; Ottino 1989).

The developments of the last decades in chaotic advection led to an advanced understanding supported by a number of laboratory experiments (e.g., Sommerer and Ott 1993; Sommerer et al. 1996; Mathur et al. 2007).
The concepts have found wide applications in geophysical and oceanographic contexts as Ruppert-Felsot et al. (2005), Shadden et al. (2005, 2009), Kai et al. (2009), and Peacock and Haller (2013) illustrate.

In dynamical systems theory, topological entropy is a measure of the complexity of the motion. In the most abstract setting, this quantity characterizes how the number of possible trajectories grows in time, in terms of an appropriately defined symbolic encoding (Ott 1993). The concept is most clearly accessible in periodically driven cases, where a slightly different formulation is based on the fact that there exist unstable periodic orbits-so-called cycles-available for the dynamics. The temporal length of these cycles can be arbitrarily large. Moreover, the number $N_{t}$ of all the unstable cycles of length $t$ increases drastically, exponentially for large times $t$. The growth rate $h$, defined by the relation $N_{t} \sim e^{h t}$, is called the topological entropy. The definition is motivated by Boltzmann's relation $S=k_{B} \ln N$ known from statistical physics, where $N$ is the number of states, $S$ is the thermodynamical entropy, and $k_{B}$ is Boltzmann's constant. It can be seen that the equivalent of $S$ is $h t ; h$ is thus some kind of entropy density.

The existence of $h$ is a basic property of chaos. A possible definition of chaos is "a system is chaotic if its topological entropy is positive" (Ott 1993; Tél and Gruiz 2006). The unstable cycles form the skeleton of chaos; chaotic motion can be considered as a random walk among the unstable cycles. The motion might temporarily approach one of the cycles. Since, however, the cycle is unstable, the trajectory can only remain in its neighborhood for a finite time and it approaches another one sooner or later. This is the origin of the irregular nature of chaotic dynamics. Consequently, chaotic motion does not repeat itself: it cannot be decomposed into the sum of even infinite number of periodic motions with discrete frequencies.

A property of topological entropy that is easier to capture in measurements is that it is also the growth rate of the length of line segments. A line segment of initial length $L_{0}$ is stretched more and more in the unstable direction of the dynamics. Let $L(t)$ denote the length of the line segment after time $t$. (Note that the line segment will always remain a one-dimensional object.) For two-dimensional systems, it is proven (Newhouse and Pignataro 1993) that after a sufficiently long time this length increases exponentially, and the growth rate is given by just $h$, according to the relation

$$
L(t) \sim e^{h t},
$$

valid for $t \gg 1 / h$. Qualitatively speaking, in the course of its stretching, the line segment approaches more and 
more unstable cycles, and in time $t$ each orbit of length $t$ gives an approximately identical contribution to its growth. This property has numerically been demonstrated by investigating the growth of material lines in laminar time periodic flows, such the von Kármán vortex street (Lai and Tél 2011; Ziemniak et al. 1994).

The topological entropy is similar in spirit to, but different in value from, the (largest positive) Lyapunov exponent $\lambda$. A general inequality states (Ott 1993; Tél and Gruiz 2006) that

$$
h \geq \lambda \text {. }
$$

The difference lies in the fact that though the Lyapunov exponent is the rate of deviation between nearby trajectories, a detailed investigation of its basic definition reveals that the linear growth rate of the logarithm of the distance between a particle pair should be determined. In contrast, $h$ is the rate of change of a length (and not of its logarithm). Inequality (2) is a consequence of the mathematical property that the logarithm of the average of a quantity is not the same as the average of the logarithm of the same quantity (known as Jensen's inequality for convex functions).

Within the realm of dynamical systems theory, there is an increasing interest in the effect of nonperiodic drivings. Recent theories (Lai and Tél 2011) claim that most of the problems in this class can be understood within the framework of so-called random maps, and the features characterizing the periodically driven case remain practically valid. In particular, the role of strictly periodic unstable orbits is taken over by unstable bounded orbits that are not exactly periodic, but that closely return to their original initial position with different return times. The number of these unstable orbits is also proliferating with the length of return time in an exponential manner, and the concept of topological entropy holds. It is in this spirit that we expect the length of material line to grow according to Eq. (1) also in turbulencedriven chaotic advection. We consider this equation to be a practical definition of the topological entropy in this case.

Technically, the evaluation of the topological entropy requires the monitoring of a large number of particles. This difficulty is, however, compensated by the fact that no smallness requirement or reshifting conditions are to be fulfilled [the latter are needed (Ott 1993) for the Lyapunov calculation since the distance between the pair should always remain small]. In particular, in flows represented on a grid, as in our case (see section 3), the determination of the Lyapunov exponent faces the difficulty of being forced to small scales. The determination of the topological entropy is based, however, on lengths exceeding by far the grid scale. The stretching filaments foliate regions with considerably different wind fields and are, therefore, natural candidates for providing a global characteristic of the atmosphere. Altogether, our experience shows that the numerical determination of the topological entropy appears to be straightforward and computationally rather cheap.

\section{Equations, data, and methods}

The motion of a tracer particle of negligible size is determined by the constraint that the particle takes on the wind velocity instantaneously. The equation of motion can then be written in the form

$$
\dot{\mathbf{r}}_{p}(t) \equiv \mathbf{v}_{p}(t)=\mathbf{v}\left(\mathbf{r}_{p}, t\right)
$$

where $\dot{\mathbf{r}}_{p}$ is the velocity of the particle and $\mathbf{v}\left(\mathbf{r}_{p}, t\right)$ is the velocity of the ambient air at the location $\mathbf{r}_{p}$ of the particle at time $t$. The solution of this equation is the path or trajectory $\mathbf{r}_{p}(t)$ of the tracer.

Equation (3) represents a dynamical system, and as such, it possesses topological entropy. This implies that topological entropy can be determined for any flow, in whatever format the flow is given. Topological entropy has thus recently been proven to be useful in oceanographic context (Thiffeault 2010), where typical values are more than an order of magnitude smaller than in the atmosphere.

It also follows from Eq. (3) that a trajectory, and hence the topological entropy, is a Lagrangian concept. The velocity field itself is Eulerian - it refers to a property in which the identity of fluid elements is not important. In contrast, Lagrangian quantities are concerned with the identity of individual fluid elements. They track the changing individual properties along the paths of the fluid elements as they are advected by the flow. Lagrangian quantities are thus integrals of Eulerian ones over time intervals and cannot therefore simply be related to Eulerian quantities taken at preselected time instants. A trajectory itself is an integral over the velocity field at locations the particle passes by over its history. In addition, Eq. (3) also indicates that the topological entropy is a quantity that is based on the velocity field solely. Temperature, humidity, and others have thus no direct influence on topological entropy.

The meteorological fields used for the simulations are given on pressure levels; therefore, we determine trajectories in pressure coordinates. Since in the horizontal direction spherical coordinates are used, we solve the following equations to determine the position of the particles: 

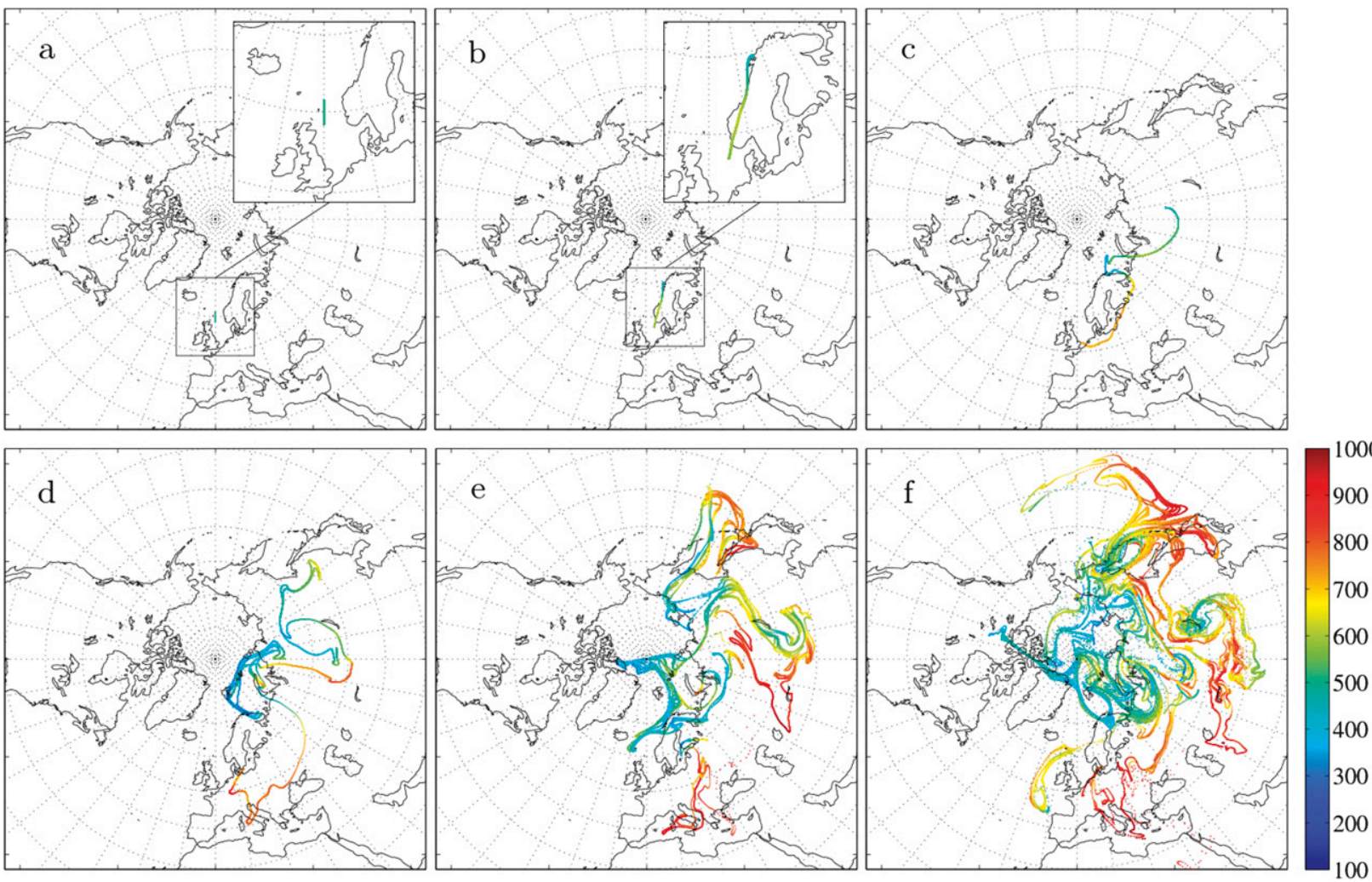

FIG. 1. Dispersion of a line segment consisting of $n=2 \times 10^{5}$ particles initialized at 0000 UTC 1 Jun 2010 . Initial conditions: $\lambda_{0}=0^{\circ}, \varphi_{0}=$ $60^{\circ} \mathrm{N}, p_{0}=500 \mathrm{hPa}, L_{0}=3^{\circ} \approx 333 \mathrm{~km}$, and the tracers are distributed uniformly along a meridian. Location of the particles (a) 0 , (b) 2 , (c) 4, (d) 6, (e) 8, and (f) 10 days after the "emission." Color bar indicates the pressure level (hPa) of the particles.

$$
\begin{aligned}
& \frac{d \lambda_{p}}{d t}=\frac{u\left(\lambda_{p}, \varphi_{p}, p_{p}, t\right)}{R_{E} \cos \varphi_{p}}, \\
& \frac{d \varphi_{p}}{d t}=\frac{v\left(\lambda_{p}, \varphi_{p}, p_{p}, t\right)}{R_{E}}, \\
& \frac{d p_{p}}{d t}=\omega\left(\lambda_{p}, \varphi_{p}, p_{p}, t\right),
\end{aligned}
$$

where $\lambda_{p}$ and $\varphi_{p}$ are the longitude and latitude coordinates of a particle; $p_{p}(t) \equiv p\left[\mathbf{r}_{p}(t), t\right]$ is the pressure coordinate of a particle along its path; $u, v$, and $\omega$ are the components of the wind field in these coordinates; and $R_{E}$ is the radius of Earth.

The particle trajectories are computed using the $u, v, \omega$ reanalysis fields (1 December 2009-30 November 2010) of the Interim European Centre for MediumRange Weather Forecasts (ECMWF) Re-Analysis (ERAInterim) database (Dee et al. 2011). The meteorological variables are available at 22 pressure levels between 1000 and $100 \mathrm{hPa}$ on a $1.5^{\circ} \times 1.5^{\circ}$ horizontal grid with $6 \mathrm{~h}$ of time resolution.
To compute trajectories, the wind data on the regular grid are interpolated to the location of the particles (using bicubic spline interpolation in horizontal and linear interpolation in vertical directions and in time). The equations of motion [Eqs. (4a)-(4c)] are solved by Euler's method with a time step of $\Delta t=45 \mathrm{~min}$. We chose this time step because by reducing it further, no notable changes in the dispersion of particles could be found.

\section{A case study}

Figure 1 illustrates the dispersion of an initially meridional line segment of $n=2 \times 10^{5}$ particles with $L_{0}=$ $3^{\circ} \approx 333 \mathrm{~km}$ (twice the resolution of the wind data) for 10 days. The center of mass of the line segment is located at $\lambda_{0}=0^{\circ}, \varphi_{0}=60^{\circ} \mathrm{N}$, and at a height of $p_{0}=500 \mathrm{hPa}$.

In the first days, the particles are advected northeast toward the Scandinavian Peninsula (Fig. 1b), while the length of the filament increases. On the fourth day (Fig. 1c), the middle part of the filament is captured by a cyclone over Finland, while the easterly end also begins to 


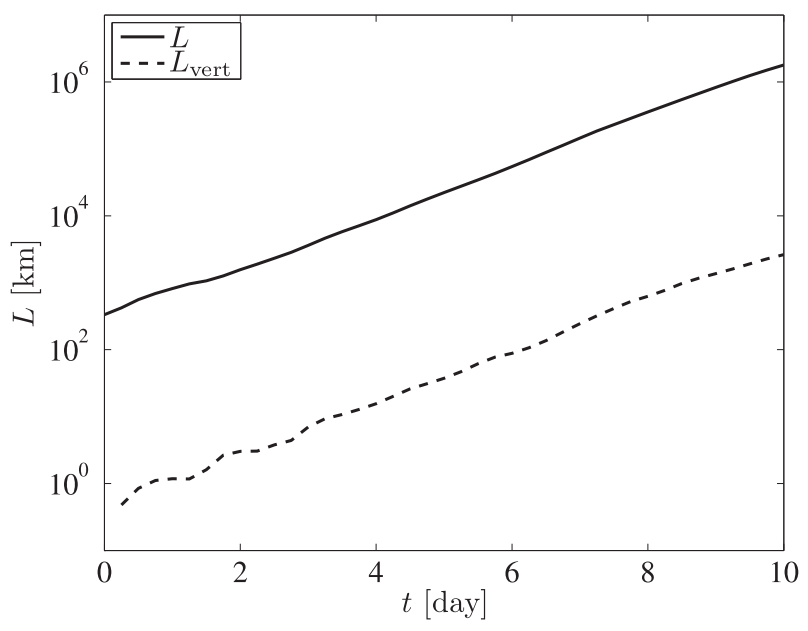

FIG. 2. The growth of the length of the line segment in Fig. 1. Here $L$ and $L_{\text {vert }}$ denote the length [Eq. (7)] and the length obtained from the vertical differences, respectively. The growth rates happen to coincide.

spiral around another cyclone over Siberia. During the next few days (Figs. 1d-f), the cyclones and anticyclones of the atmospheric flow fold, rumple, and lengthen the filament more and more by stirring, and at the end of the observation period, the extent of the line segment becomes several thousand times greater than the initial length (Fig. 2) and extends over Europe and quite a large area of Asia. It is also interesting, as shown in Fig. $1 \mathrm{f}$, that after 10 days the altitude of the particles spans to a wide range between about 300 and $1000 \mathrm{hPa}$.

The algorithm of determining the length of a filament is based on the observation that initially neighboring particles remain neighbors for all times. The horizontal distance $(\mathrm{km})$ between two neighboring particles is computed along a great circle:

$$
\begin{aligned}
\left|\mathbf{r}_{p, i}-\mathbf{r}_{p, i+1}\right|_{\text {hor }}= & \arccos \left[\sin \varphi_{p, i} \sin \varphi_{p, i+1}\right. \\
& \left.+\cos \varphi_{p, i} \cos \varphi_{p, i+1} \cos \left(\lambda_{p, i}-\lambda_{p, i+1}\right)\right] \\
& \times \frac{180}{\pi} \times 111.1 .
\end{aligned}
$$

Here, $\lambda_{p, i}$ and $\varphi_{p, i}$ denote the horizontal location of particle $i$. The vertical distance is determined using the hydrostatic approximation:

$$
\left|\mathbf{r}_{p, i}-\mathbf{r}_{p, i+1}\right|_{\mathrm{vert}}=(\rho g)^{-1}\left|p_{p, i}-p_{p, i+1}\right|,
$$

where $\rho$ and $g$ are the density of the air and the gravitational acceleration, respectively. We note that deviations from the hydrostatic approximation are known to be negligible on length scales larger than about $30 \mathrm{~km}$ (e.g., Kalnay 2003), with the typical spatial resolution of $100 \mathrm{~km}$ of the database used here hydrostatic relations are thus applicable. Since we shall see that vertical displacements are not important, for simplicity, calculations have been carried out with a constant average air density of $\rho=0.7 \mathrm{~kg} \mathrm{~m}^{-3}$.

Stretching in the vertical direction is negligible compared to the horizontal one. The proportion is found to be about $10^{-2}-10^{-3}$ (Fig. 2); therefore, in calculating the distance $\left|\mathbf{r}_{p, i}-\mathbf{r}_{p, i+1}\right| \cong\left(\left|\mathbf{r}_{p, i}-\mathbf{r}_{p, i+1}\right|_{\text {hor }}^{2}+\left|\mathbf{r}_{p, i}-\mathbf{r}_{p, i+1}\right|_{\text {vert }}^{2}\right)^{1 / 2}$ between two particles the second term in parentheses can be neglected.

The length of the filament at time $t$ is computed as the sum of the horizontal distances between neighboring particle positions $\mathbf{r}_{p, i}$ at time $t$ :

$$
L(t)=\sum_{\substack{i=1 \\ p_{p, i}<p_{s}}}^{n-1}\left|\mathbf{r}_{p, i}(t)-\mathbf{r}_{p, i+1}(t)\right|_{\text {hor }} .
$$

In Eq. (7) $p_{p, i}<p_{s}\left(p_{s}=1000 \mathrm{hPa}\right)$ expresses that only those particles contribute to the length of the filament that remain in the atmosphere. ${ }^{1}$

It is clearly visible in Fig. 2 that the growth of $L(t)$ is exponential in time. The exponent, the topological entropy, is found to be $h=0.89 \mathrm{day}^{-1}$. The error of $h$ is determined from the uncertainty of the least squares fittings to different time intervals within days $2-10$, and is found to be $\pm 0.02 \mathrm{day}^{-1}$. For completeness, we also determined $L_{\mathrm{vert}}(t)$, the sum of the modulus of the vertical distances of all neighboring particle pairs. This quantity is found to grow with the same exponent $h$. The ratio $L_{\text {vert }}(t) / L(t)$ is approximately $1.5 \times 10^{-3}$, which supports the view that the total length is determined basically by the horizontal one.

The value of $h=0.89$ day $^{-1}$ in this case study implies that the total stretching factor after 10 days is $\exp (8.9) \approx$ 7330. The average initial distance $L_{0} / n=3 \%\left(2 \times 10^{5}\right)=$ $1.5 \times 10^{-5 \circ} \approx 1.7 \mathrm{~m}$ of neighboring pairs has thus grown to $7330 \times 1.7 \mathrm{~m}=12.5 \mathrm{~km}$. This is much smaller than the total horizontal extent of the filament, which justifies a posteriori the use of the simple approximation in Eq. (7) to determine the distance.

\footnotetext{
${ }^{1}$ To enlarge the temporal range where a clear exponential can be fitted to the graph of $L(t)$, we have extended the validity of Eq. (3) down to the surface. We have found that even passive tracers reach the surface within the observed time span of 10 days. The proportion of the outfalling particles in the simulations is, however, small—usually less than $0.1 \%$. These few particles have been taken out from the simulation, and neighborhood relations have been reconsidered: particles between which a particle has left the atmosphere become neighbors in the next time step.
} 


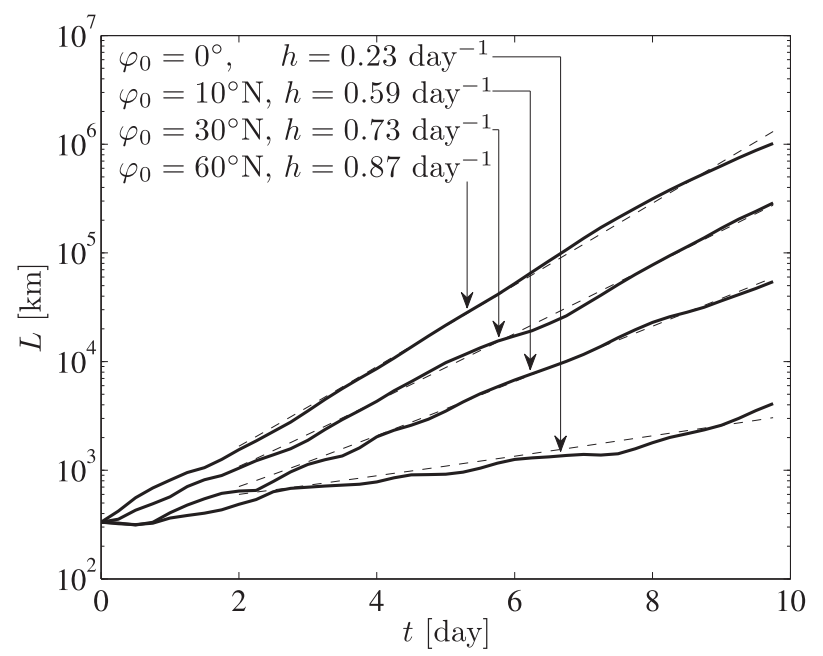

FIG. 3. Dependence on the initial latitude. The growth of $L(t)$ of meridional line segments (consisting of $n=2 \times 10^{5}$ particles) initialized at 0000 UTC 1 Apr 2010 at $\lambda_{0}=0^{\circ}, \varphi_{0}$ (indicated in the legend), and $p_{0}=500 \mathrm{hPa}$. The initial length of the line segments is $L_{0}=3^{\circ} \approx 333 \mathrm{~km}$. The $h$ values in the legend are obtained from the slopes of the dashed lines.

\section{Geographical and seasonal changes}

Figure 3 illustrates that the stretching rate of line segments started at different latitudes at the same time varies significantly. The shortest total length is found at the equator $\left(h=0.23 \mathrm{day}^{-1}\right)$, while the longest filament is formed from the one located at $60^{\circ} \mathrm{N}\left(h=0.87\right.$ day $\left.^{-1}\right)$ initially.

The relatively strong location dependence motivated us to determine the geographical distribution of the topological entropy. We initialize material line segments oriented meridionally over the globe. The initial height is $p_{0}=500 \mathrm{hPa}$ for all the particles. These segments are distributed over the globe on a grid: from $80^{\circ} \mathrm{S}$ to $80^{\circ} \mathrm{N}$ in $10^{\circ}$ increments, and starting at $180^{\circ}$ longitude in $30^{\circ}$ increments. The topological entropy of each line segment is calculated from a 10-day tracking. To gain an annual cycle of the topological entropy, at each geographical location a line segment is initialized in every 10 days, and then the temporal average of the topological entropy of 3 months is determined for December-February, March-May, June-August, and September-November (Fig. 4). The largest values appear in the mid- and high latitudes, mainly in the winter season of the hemisphere because of the strong mixing and shearing effects of cyclones. So we can find the largest topological entropies in December-February in the Northern Hemisphere (Fig. 4a) and in June-August in the Southern Hemisphere (Fig. 4c).

Based on Fig. 4, we also determined the zonally averaged $h$ as a function of $\varphi$ (Fig. 5). The zonally and seasonally averaged topological entropy in the mid- and high latitudes $\left(30^{\circ}-80^{\circ}\right)$ in the winter season of the hemispheres is somewhat larger than in the summer
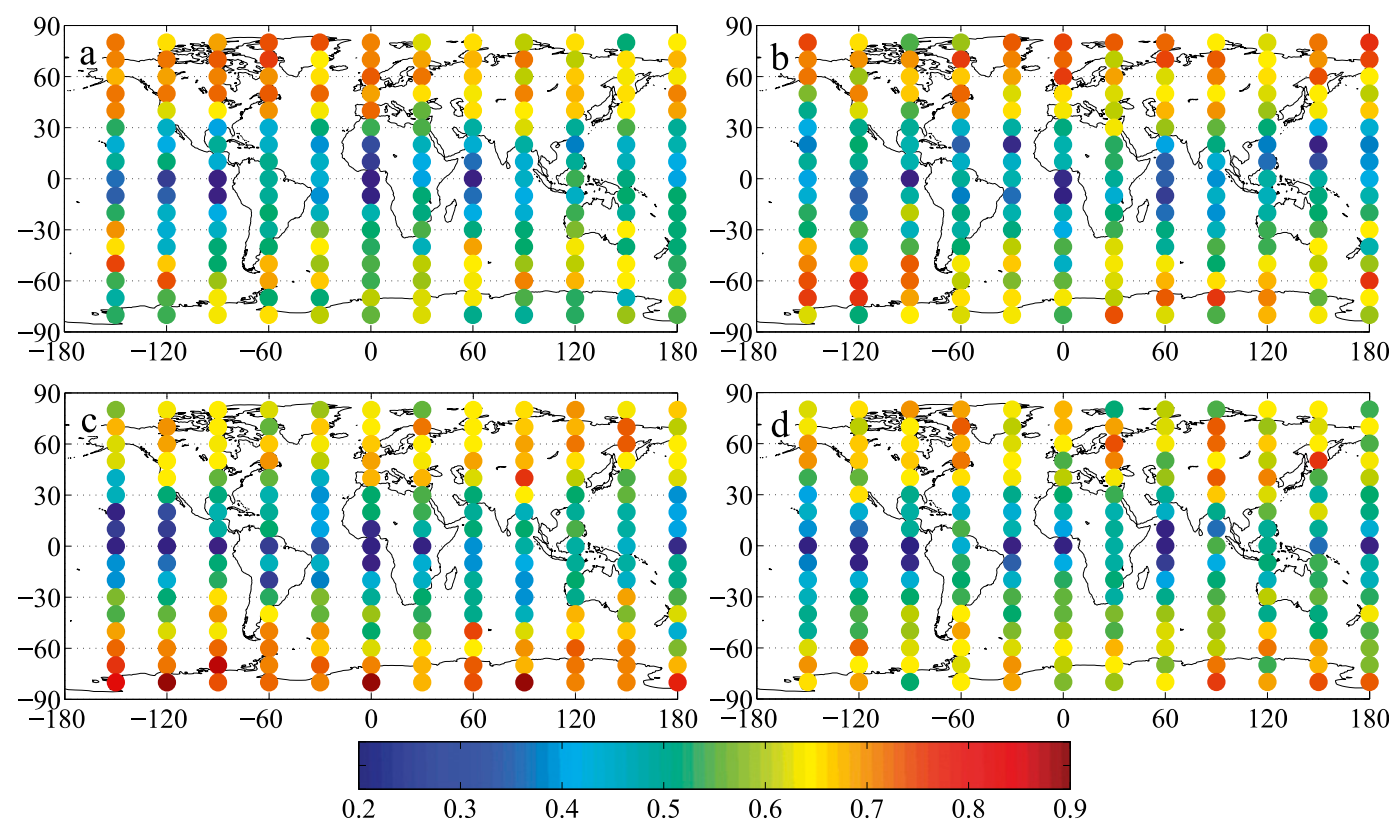

FIG. 4. Geographical distribution of the average seasonal topological entropy $\left(\right.$ day $\left.^{-1}\right)$ obtained from line segments (consisting of $n=2 \times 10^{5}$ particles) initiated on the $p_{0}=500$-hPa level in (a) December-February, (b) March-May, (c) June-August, and (d) September-November. The initial length is $L_{0}=3^{\circ}$, and the center of mass of the meridional segments is at the midpoints of the colored discs. 

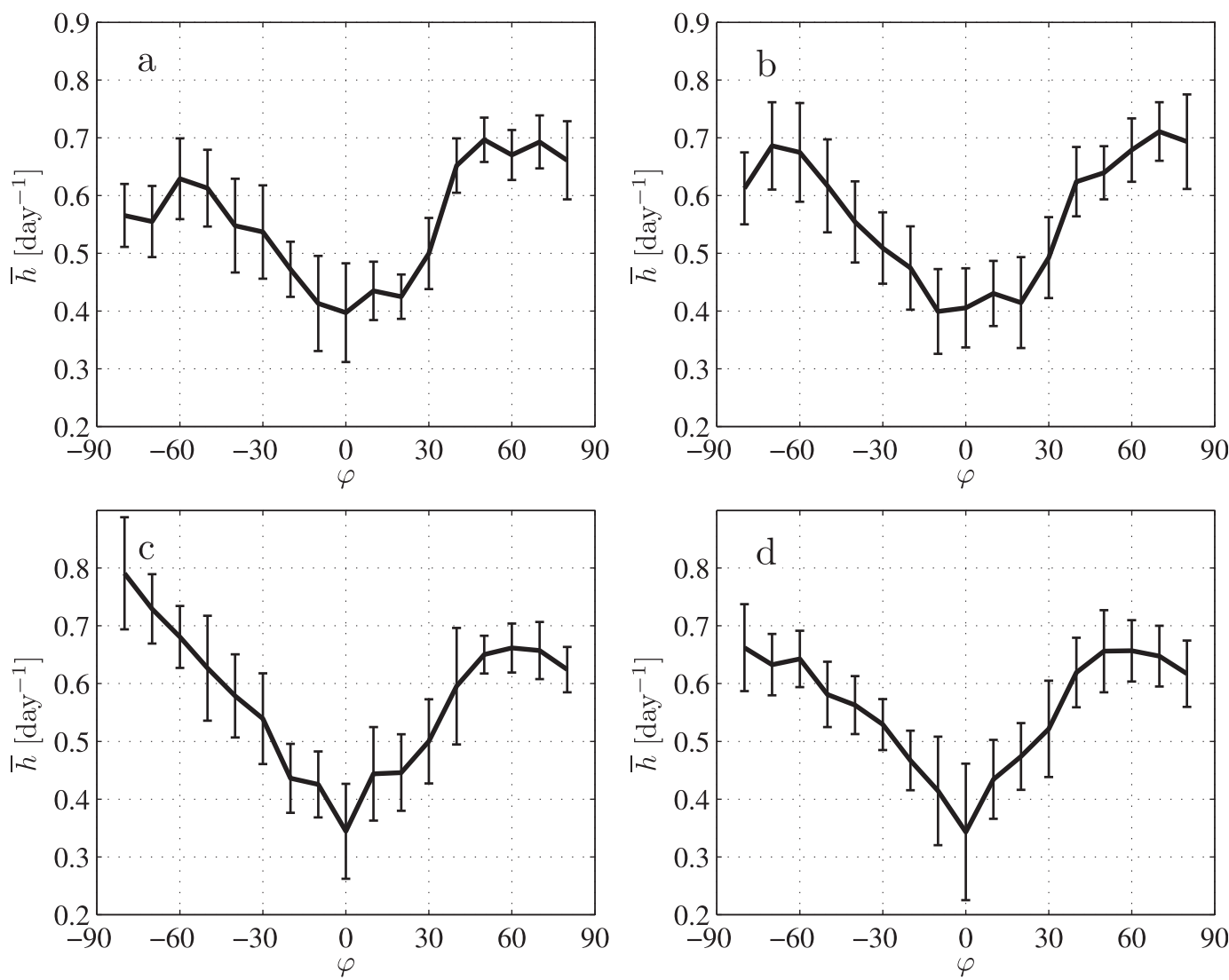

FIG. 5. Latitudinal distribution of the zonally and seasonally averaged topological entropy obtained from line segments (consisting of $n=2 \times 10^{5}$ particles) initiated on the 500-hPa level in (a) December-February, (b) MarchMay, (c) June-August, and (d) September-November. The initial length is $L_{0}=3^{\circ}$. The error bars represent the standard deviation of the $h$ values in Fig. 4 along a line of latitude.

$\left(\bar{h}_{w}-\bar{h}_{s} \approx 0.06 \mathrm{day}^{-1}\right)$. This is in agreement with the fact that winters are more variable than summers because of the greater temperature gradient between the pole and the equator. The difference between the winter and summer seasons is more significant in the Southern Hemisphere than in the Northern Hemisphere ( 0.09 and 0.04 day $^{-1}$, respectively). The reason for this can be the difference in the proportion and location of oceans and continents. In March-May (Fig. 5b) and in SeptemberNovember (Fig. 5d) there are no great differences between the two hemispheres; however, March-May seems to be a more intense period in both hemispheres based on the average seasonal topological entropy data. The mean error of the estimator $h$ on this global level is found to be $2.5 \%$.

\section{Local variances and relevance to spreading of contaminants}

There might be considerable deviations from the topological entropy averages given above, and these might indicate basically different scenarios of dispersion. To illustrate this, Fig. 6 shows that significant topological entropy differences can be present in the tropics. Although the mean topological entropy here is about 0.4 day $^{-1}$, at certain locations the length of the filament increases much slower or faster. Figure 6a demonstrates a weak dispersion: the particles initiated close to Ecuador travel with the trade winds while they happen to sink. The topological entropy of the filament is small: $h=0.25$ day $^{-1}$. However, in Fig. $6 \mathrm{~b}$ the particles distributed around Papua New Guinea rise high in a low-pressure system (which formed on 3 January and dissipated on 8 January), and the filament becomes strongly folded in the upper levels of the atmosphere. In this case the topological entropy is $h=0.58 \mathrm{day}^{-1}$. We thus see that a value of $h$ around 0.3 hardly represents any danger for a strong spreading of a pollutant over 10 days, while twice of this value implies a considerable risk for a convoluted dispersion over an extended area after the same time interval.

Besides the topological entropy, the fractal dimension of the line segments in Figs. 1, 6a, and $6 \mathrm{~b}$ is also 

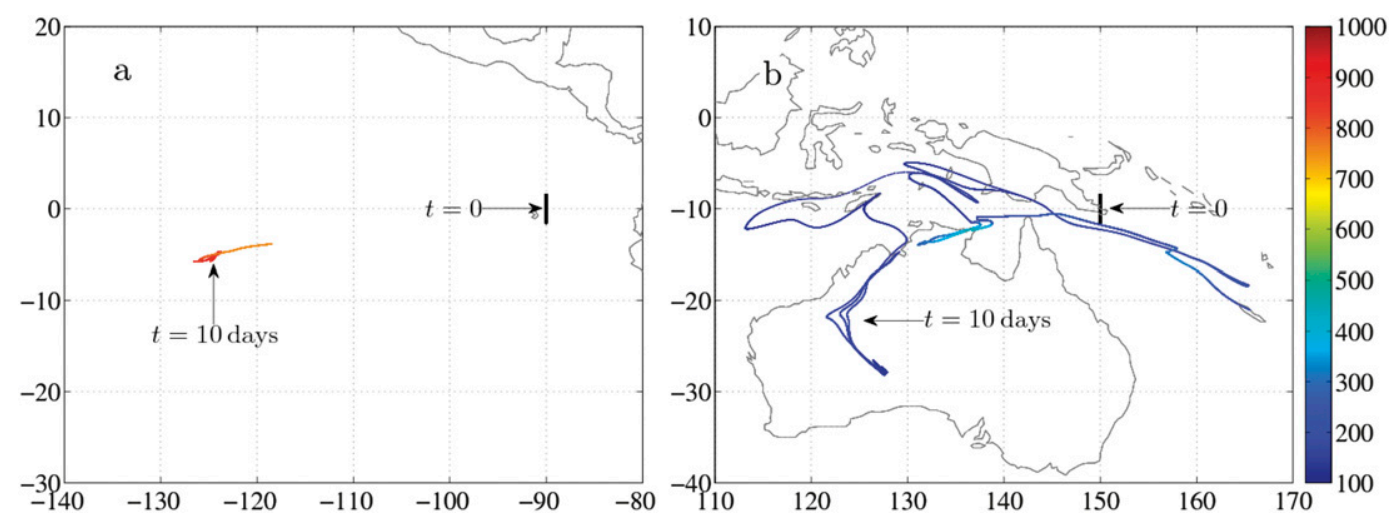

FIG. 6. The distribution of $n=2 \times 10^{5}$ particles of a meridional line segment initialized at 0000 UTC 1 Jan 2010 (black line) after 10 days (colored line). Initial conditions: (a) $\lambda_{0}=90^{\circ} \mathrm{W}, \varphi_{0}=0^{\circ}, p_{0}=500 \mathrm{hPa} ;(\mathrm{b}) \lambda_{0}=150^{\circ} \mathrm{E}, \varphi_{0}=$ $10^{\circ} \mathrm{S}, p_{0}=500 \mathrm{hPa}$. The initial length is $L_{0}=3^{\circ}$ in both cases. Color bar indicates the pressure level (hPa) of the particles.

estimated in each time step of the simulation (see Fig. 7). In fact, these figures have been redone with 10 times more particles than originally. Fractal dimension $D_{0}$ is determined by using the formula $N(\varepsilon) \sim \varepsilon^{-D_{0}}$, where $N(\varepsilon)$ denotes the number of horizontal cells of characteristic size $\varepsilon$ that cover the entire line segment. Instead of cells of a regular latitude-longitude grid (where, e.g., a $0.5^{\circ} \times 0.5^{\circ}$ cell has much smaller area near the pole than near the equator), we design here an alternative grid (see Fig. 7a) constructed in the following way: the meridional sides of a cell is $\Delta \varphi=\varepsilon$ for all latitudes, while the zonal sides $\Delta \lambda$ of the cells vary with latitude so that the area of the cells is almost equal (a belt of a latitude has to be filled with integer number of cells; therefore, the area of the cells at different latitudes differ only slightly). At the equator $\Delta \lambda=\varepsilon$. The fractal dimension is estimated by calculating $N(\varepsilon)$ at different times and fitting a straight line to the $\ln N$ versus $\ln (1 / \varepsilon)$ graph over an interval of 1.5 decades, within the range of $\varepsilon=0.025^{\circ}-10^{\circ}$, where a clear linear behavior can be seen. The error of $D_{0}$ is obtained as the error of the least squares fitting over this interval.

Figure $7 \mathrm{~b}$ shows the dimensions over 30 days. Note that all three line segments remain on average horizontal lines, indicating $D_{0} \approx 1$, over the first 5 days. As the material lines stretch and fold, and their shape becomes more complex, the fractal dimensions begin to increase. The growth is the fastest for Fig. 1 and the slowest for Fig. 6a, which is in harmony with the visual impression gained from these figures. After 10 days, the
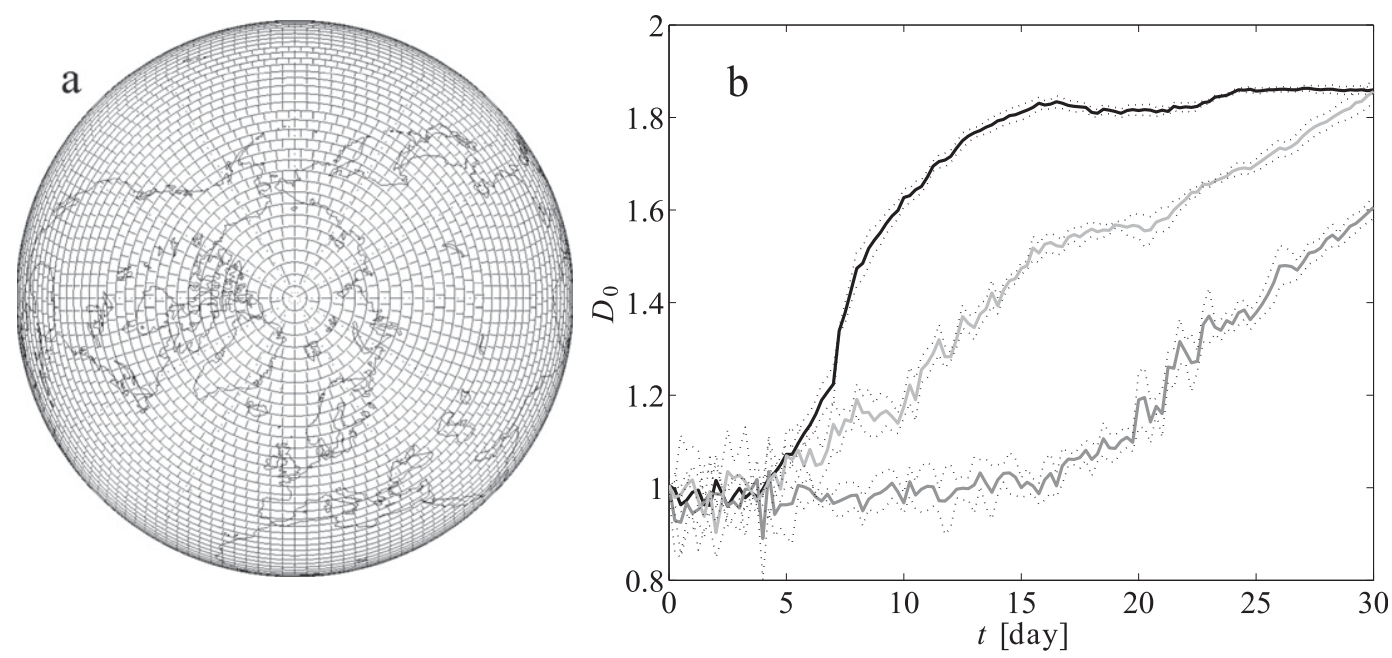

FIG. 7. (a) The nearly uniform grid used for the determination of the fractal dimension. Here $\varepsilon=1.5^{\circ}$. (b) Fractal dimension of filaments initiated as meridional line segments in Figs. 1 (black), 6a (dark gray), and 6b (light gray). Dots represent the error bar at a given value of $D_{0}$. 


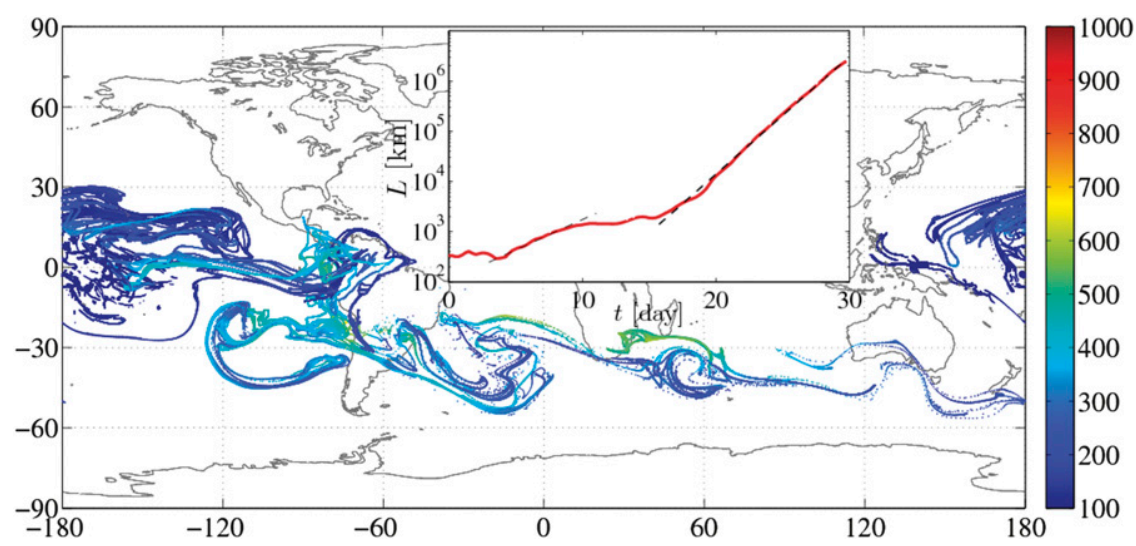

FIG. 8. The distribution of $n=2 \times 10^{6}$ particles at the end of day 30, initiated on the meridional line segment of Fig. 6a. Color bar indicates the pressure level (hPa) of the particles. The inset shows the material length over 30 days. In the period 10-15 days, a crossover takes place from $h=0.25 \mathrm{day}^{-1}$ to a larger value of the topological entropy $h=0.54 \mathrm{day}^{-1}$.

fractal dimension of the latter is still $D_{0} \approx 1$, but that of Fig. $6 \mathrm{~b}$ is $D_{0} \approx 1.2$. The object with several foldings in Fig. 1 is characterized by $D_{0} \approx 1.6$. All dimensions intend to grow farther in the next 20 days and are expected to converge to 2 asymptotically.

Qualitatively, more convoluted shapes imply larger dimensions. The dimension can thus be used as a measure of the geographical extension of the risk or of the need of evacuation from a large territory. In particular, over the first 5 days, while the dimension is unity, the extension of the infected region grows as $L(t)$. Hence, the size of the infected region also grows exponentially in time, with the topological entropy as the rate. (The growth of the infected area then slows down in time since the full hemisphere, or globe, will be filled in asymptotically.)

\section{Discussion and outlook}

As a continuation of the remark of the last paragraph, we mention that the full atmosphere is a closed system, and closed, well-mixed dynamical systems are characterized by a single value of the topological entropy (Ott 1993). In our case the time over which this location- and time-independent value is expected to show up is the time needed for a more or less uniform spreading over both atmospheres. This is on the order of several months (e.g., Haszpra et al. 2012), and the time span used here ( $\sim 10$ days) is much shorter. To illustrate that the growth rate of the length changes on time scales longer than 10 days, we determine the length in one of our case studies (see Fig. 6a) up to 30 days with an enlarged particle number. As the inset of Fig. 8 shows, a new, steeper part appears at longer times and the slope there $\left(h=0.54\right.$ day $\left.^{-1}\right)$ is closer to those characteristic to the midlatitudes. This can be considered as a signature of the convergence of the topological entropy to a universal value for long times. The spread of the particles after 30 days, shown in Fig. 8, illustrates that they are in the process of approaching a well-mixed state over the southern and northern midlatitudes. The topological entropy used throughout the paper is thus a kind of finite time entropy. We have chosen the time span of about 10 days since this appears to be the shortest, but a sufficiently long time interval over which a clear exponential growth of the length can be observed.

Subgrid-scale motions are known to have an impact on particle dispersion. To see an example of how these might influence the results of topological entropy calculations, we choose parameterizing turbulence. To this end, we use a Lagrangian random-walk formulation for turbulent diffusion and extend Eq. (3) to a stochastic differential equation for each particle with turbulent diffusivity $\mathbf{K}=\left(K_{h}, K_{h}, K_{v}\right)$ as a diffusion coefficient. The equations of motion are similar, for example, to those of the atmospheric dispersion model GEARN (Terada and Chino 2008). As a result, infinitesimally close particles can jump far away from each other within a time step; therefore, the spatial neighborhood relations of the particles [discussed above Eq. (5)] typically break down. The material line becomes locally very much zigzagged. In the simulations, therefore, we find natural to consider the "coarse grained" horizontal length obtained by counting the number of boxes of a given size that cover the set of particles at a time instant. Of course, the length depends on the box size: toolarge boxes, for example, cannot capture the small-scale filaments. The slope of the coarse-grained lengths (i.e., the value of the topological entropy) is found, however, to not depend on the box size in the range $0.025^{\circ}-0.5^{\circ}$. 


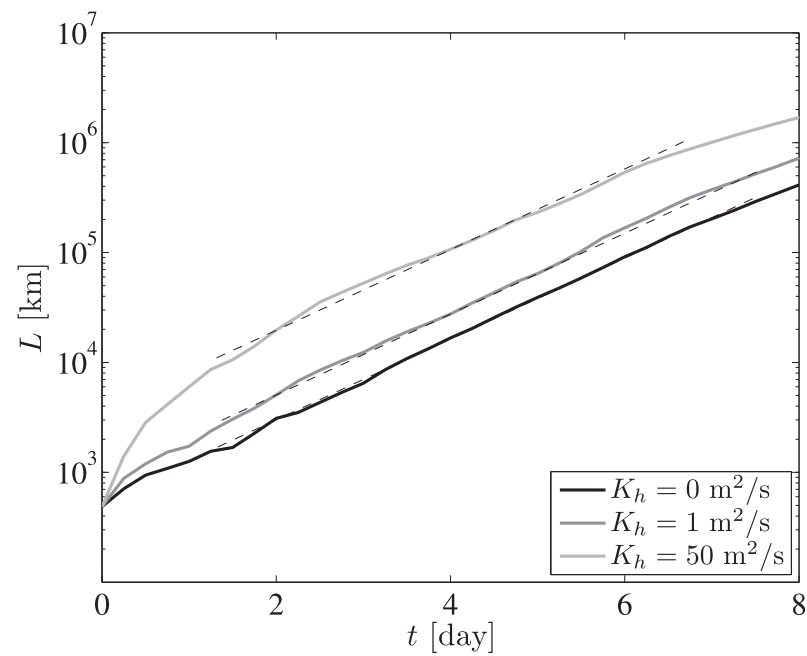

FIG. 9. The growth of the coarse-grained length (obtained with a box size $0.1^{\circ}$ ) of the line segment initialized in Fig. 1 including constant horizontal turbulent diffusivity $K_{h}$ (values indicated in the legend). To guide the eyes, dashed lines represent slopes of $0.87 \mathrm{day}^{-1}$, the topological entropy of the diffusionless case.

The curves in Fig. 9 illustrate the growth of the coarse grained length obtained with box size $0.1^{\circ}$ for the situation of the case study of Fig. 1 in the presence of a constant horizontal turbulent diffusivity $K_{h}=1$ and $50 \mathrm{~m}^{2} \mathrm{~s}^{-1}\left(K_{v}=0 \mathrm{~m}^{2} \mathrm{~s}^{-1}\right)$. As expected, the larger the diffusivity the larger the length at a given time instant. Figure 9 demonstrates that the topological entropy seems to hardly change in the presence of horizontal diffusion. (The topological entropy of the diffusionless case obtained by this method is $h=0.87 \pm 0.03 \mathrm{day}^{-1}$, which is consistent with the one in section 4 in view of the error bars.) Note that diffusivity shortens the range over which a clean exponential growth can be seen.

In another run, we take into account also vertical diffusivity $K_{v}$. It is calculated only within the boundary layer using Monin-Obukhov similarity theory utilizing the time-dependent boundary layer height, sensible heat flux, temperature, and surface stress data (Holtstag and Boville 1993). The new version of Eq. (4c) includes then a stochastic term of $K_{v}$, similar to that in the horizontal direction, but, in addition, a new, nonstochastic drift term also appears containing the vertical gradient of $K_{v}$. Results (not shown) reveal that the impact of the vertical diffusivity hardly modifies the values of the topological entropy on large scales obtained without $K_{v}$ for particles initiated in the free atmosphere.

Besides these preliminary studies, a more detailed analysis remains for future work to explore how nongeostrophic effects such as frontal areas and strong convective storms taken place well below the grid size of reanalysis data can be parameterized. The use of finer databases, or wind-field forecasts, would also be worthwhile. It also remains to be seen what the best spatially smooth parameterization of subgrid turbulence would be for topological entropy studies.

A recent theoretical development suggests regarding particle trajectories as braids and determines topological entropy based on results taken from braid theory. The method leads to a new interpretation of the topological entropy as a measure of the entanglement of trajectories. Interestingly, even a low number of trajectories appears to be sufficient for a proper estimation of the entropy. This was applied to the ocean (Thiffeault 2010) and might also be useful in the atmospheric context. Furthermore, topological entropy and braid theory have recently been suggested (Allshouse and Thiffeault 2012; Thiffeault 2010) to be efficient tools to determine Lagrangian coherent structures (Shadden et al. 2009; Peacock and Haller 2013).

We mention in closing that a similar analysis can be carried out with aerosol particles, too [for a preliminary result, see Haszpra and Tél (2011)]. In this case, however, the topological entropy does not reflect merely the state of the atmosphere; its value can depend on the properties of the aerosol particles. Such simulations might be useful to see the dynamics of the spreading contamination if the pollution consists of aerosol particles, such as in the case of nuclear accidents and volcanic eruptions.

Acknowledgments. The authors thank the useful remarks of the anonymous reviewers and G. Drótos, J.-L. Thiffeault, and G. Wotawa for useful suggestions. V. Homonnai, A. Horányi, T. Horváth, I. M. Jánosi, L. I. Lagzi, and M. Vincze are also acknowledged for valuable comments and remarks. This work was supported by the Hungarian Science Foundation under Grant OTKA NK100296, by EU COST Action MP0806 on "Particles in Turbulence," by the European Union and the European Social Fund through project FuturICT.hu (Grant TAMOP- 4.2.2.C-11/1/KONV2012-0013), and by the von Humboldt Foundation.

\section{REFERENCES}

Allshouse, M. R., and J.-L. Thiffeault, 2012: Detecting coherent structures using braids. Physica D, 241, 95-105.

Aref, H., 1985: Stirring by chaotic advection. J. Fluid Mech., 143, $1-21$.

Bowman, K. P., 1993: Large-scale isentropic mixing properties of the Antarctic polar vortex from analyzed winds. J. Geophys. Res., 98 (D12), 23013-23027.

Dee, D. P., and Coauthors, 2011: The ERA-Interim reanalysis: Configuration and performance of the data assimilation system. Quart. J. Roy. Meteor. Soc., 137, 553-597. 
Haszpra, T., and T. Tél, 2011: Volcanic ash in the free atmosphere: A dynamical systems approach. J. Phys., 333, 012008, doi:10.1088/1742-6596/333/1/012008.

—_, P. Kiss, T. Tél, and I. M. Jánosi, 2012: Advection of passive tracers in the atmosphere: Batchelor scaling. Int. J. Bifurcation Chaos, 22, 1250241, doi:10.1142/S0218127412502410.

Holtstag, A. A. M., and B. A. Boville, 1993: Local versus nonlocal boundary-layer diffusion in a global climate model. J. Climate, 6, 1825-1842.

Huber, M., J. C. McWilliams, and M. Ghil, 2001: A climatology of turbulent dispersion in the troposphere. J. Atmos. Sci., 58, 2377-2394.

Kai, E., V. Rossi, J. Sudre, H. Weimerskirch, C. Lopez, E. HernandezGarcia, F. Marsac, and V. Garçon, 2009: Top marine predators track Lagrangian coherent structures. Proc. Natl. Acad. Sci. USA, 106, 8245-8250.

Kalnay, E., 2003: Atmospheric Modeling, Data Assimilation and Predictability. Cambridge University Press, 364 pp.

Lai, Y.-C., and T. Tél, 2011: Transient Chaos: Complex Dynamics on Finite Time Scales. Applied Mathematical Sciences, Vol. 173 , Springer, $410 \mathrm{pp}$.

Mathur, M., G. Haller, T. Peacock, J. Ruppert-Felsot, and H. Swinney, 2007: Uncovering the Lagrangian skeleton of turbulence. Phys. Rev. Lett., 98, 144502, doi:10.1103/ PhysRevLett.98.144502.

Mizuta, R., and S. Yoden, 2001: Chaotic mixing and transport barriers in an idealized stratospheric polar vortex. J. Atmos. Sci., 58, 2616-2629.

Newhouse, S., and T. Pignataro, 1993: On the estimation of topological entropy. J. Stat. Phys., 72, 1331-1351.

Ott, E., 1993: Chaos in Dynamical Systems. Cambridge University Press, 385 pp.

Ottino, J., 1989: The Kinematics of Mixing: Stretching, Chaos, and Transport. Cambridge Texts in Applied Mathematics, Vol. 3, Cambridge University Press, 396 pp.

Peacock, T., and G. Haller, 2013: Lagrangian coherent structures: The hidden skeleton of fluid flows. Phys. Today, 66, 41-47.

Pierrehumbert, R. T., and H. Yang, 1993: Global chaotic mixing on isentropic surfaces. J. Atmos. Sci., 50, 2462-2480.
Ruppert-Felsot, J., O. Praud, E. Sharon, and H. Swinney, 2005: Extraction of coherent structures in a rotating turbulent flow experiment. Phys. Rev., 72E, 016311, doi:10.1103/ PhysRevE.72.016311.

Shadden, S., F. Lekien, and J. Marsden, 2005: Definition and properties of Lagrangian coherent structures from finite-time Lyapunov exponents in two-dimensional aperiodic flows. Physica D, 212, 271-304.

— _ _ J. Jaduan, F. Chavez, and J. Marsden, 2009: The correlation between surface drifters and coherent structures based on high-frequency radar data in Monterey Bay. DeepSea Res. II, 56, 161-172.

Sommerer, J. C., and E. Ott, 1993: Particles floating on a moving fluid: A dynamically comprehensible physical fractal. Science, 259, 335-339.

_ - H.-C. Ku, and H. E. Gilreath, 1996: Experimental evidence for chaotic scattering in a fluid wake. Phys. Rev. Lett., 77, 5055-5058.

Stohl, A., 1998: Computation, accurancy and applications of trajectories-A review and bibliography. Atmos. Environ., 32, 947-966.

Tél, T., and M. Gruiz, 2006: Chaotic Dynamics: An Introduction Based on Classical Mechanics. Cambridge University Press, 412 pp.

Terada, H., and M. Chino, 2008: Development of an atmospheric dispersion model for accidental discharge of radionuclides with the function of simultaneous prediction for multiple domains and its evaluation by application to the Chernobyl nuclear accident. J. Nucl. Sci. Technol., 45, 920-931.

Thiffeault, J.-L., 2010: Braids of entangled particle trajectories. Chaos, 20, 017516, doi:10.1063/1.3262494.

von Hardenberg, J., K. Fraedrich, F. Lunkeit, and A. Provenzale, 2000: Transient chaotic mixing during baroclinic life cycle. Chaos, 10, 122-134.

Yang, H., and R. T. Pierrehumbert, 1994: Production of dry air by isentropic mixing. J. Atmos. Sci., 51, 3437-3454.

Ziemniak, E. M., C. Jung, and T. Tél, 1994: Tracer dynamics in open hydrodynamical flows as chaotic scattering. Physica $D$, 76, 123-146. 